\title{
Electronically Tunable Fully Integrated Fractional-Order Resonator
}

\author{
Georgia Tsirimokou, Student Member, IEEE, Costas Psychalinos, Senior Member, IEEE, Ahmed S. \\ Elwakil, Senior Member, IEEE, and Khaled N. Salama, Senior Member, IEEE
}

\begin{abstract}
A fully integrated implementation of a parallel fractional-order resonator which employs together a fractionalorder capacitor and a fractional-order inductor is proposed in this paper. The design utilizes current-controlled Operational Transconductance Amplifiers as building blocks, designed and fabricated in AMS $0.35 \mu \mathrm{m}$ CMOS process, and based on a second-order approximation of a fractional-order differentiator/integrator magnitude optimized in the range $10 \mathrm{~Hz}-700 \mathrm{~Hz}$. An attractive benefit of the proposed scheme is its electronic tuning capability.
\end{abstract}

Index Terms-Fractional-order circuits, fractional-order inductor, fractional-order resonator, fractional-order impedance.

\section{INTRODUCTION}

$\mathbf{F}$ ractional-order impedances are generalized forms of conventional circuit impedances and are essential for accurate and realistic circuit models in a wide range of applications [1]. A general electrical impedance is defined as $Z(s)=k s^{\alpha}=$

$$
(k \omega)^{\alpha}\left(\cos \left(\frac{\alpha \pi}{2}\right)+j \sin \left(\frac{\alpha \pi}{2}\right)\right)=R(\omega, \alpha)+j X(\omega, \alpha)
$$

In (1) if $(k, \alpha)=(R, 0)$ then $Z(s)$ represents a resistor while if $(k, \alpha)=(L, 1)$, then it represents an inductor (coil). For any $0<\alpha<1$, the resulting impedance is termed fractionalorder inductor (FOI), which is an inductor with frequencydependent losses. For $(k, \alpha)=(1 / C,-1), Z(s)$ represents a capacitor, while for $-1<\alpha<0$, the resulting impedance is termed a fractional-order capacitor; which is a capacitor with frequency-dependent losses [2]. A fractional-order capacitor is also known as a Constant Phase Element (CPE) due to its fixed phase angle $\theta=\left(\frac{-\alpha \pi}{2}\right)$. Note however, that there arises inconsistency in the units of $L, C$ when $\alpha \neq \pm 1$ if measured using the units of Henry or Farad respectively. The constant $k$ in (1) is thus known as pseudo-inductance and pseudo-capacitance when $\alpha \neq \pm 1$ with symbols $L_{\beta}, C_{\alpha}$ for simplicity. Therefore, a fractional-order capacitor has an impedance

$$
Z_{C_{\alpha}, \alpha}(s)=\frac{1}{C_{\alpha} s^{\alpha}}=\frac{\cos \left(\frac{\alpha \pi}{2}\right)}{\omega^{\alpha} C_{\alpha}}+\frac{\sin \left(\frac{\alpha \pi}{2}\right)}{j \omega^{\alpha} C_{\alpha}}
$$

Georgia Tsirimokou and Costas Psychalinos are with the Department of Physics, Electronics Laboratory, University of Patras, Rio Patras, GR-26504, Greece, e-mail: tsirimg@upatras.gr; cpsychal@physics.upatras.gr.

Ahmed S. Elwakil is with the Department of Electrical and Computer Engineering, College of Engineering, University of Sharjah, PO Box 27272, Emirates, e-mail: elwakil@ieee.org.

Khaled N. Salama is with the Computer, Electrical and Mathematical Sciences \& Engineering Division, King Abdullah University of Science and Technology (KAUST), Thuwal, Saudi Arabia, e-mail: khaled_salama@ieee.org. where $C_{\alpha}$ has the units of $\mathrm{Farad} / \mathrm{sec}^{1-\alpha}$. It is seen from (2) that apart from the frequency dependent resistive losses, the relationship between capacitance and pseudo-capacitance is given by [3]

$$
C=C_{\alpha} \frac{\omega^{\alpha-1}}{\sin \left(\frac{\alpha \pi}{2}\right)}
$$

In a similar way, the relationship between inductance and pseudo-inductance in a fractional-order inductor $Z_{L_{\beta}, \beta}(s)=$ $L_{\beta} s^{\beta}$ can be given by [3]

$$
L=L_{\beta} \frac{\sin \left(\frac{\beta \pi}{2}\right)}{\omega^{1-\beta}}
$$

where $L_{\beta}$ is the pseudo-inductance in units of Henry/ $\sec ^{1-\beta}$. Transformations (3) and (4) are used for the calculation of $C$ and $L$ given $\left(C_{\alpha}, \alpha\right)$ and $\left(L_{\beta}, \beta\right)$ at a center frequency of approximation $\omega=\omega_{0}$, as explained later in section III. However, to simplify the targeted circuit design, here we absorb the unit-less $\sin (\alpha \pi / 2)$ and $\sin (\beta \pi / 2)$ terms within the values of $C_{\alpha}$ and $L_{\beta}$ respectively; i.e. we employ the transformation

$$
C, L=\frac{C_{\alpha}, L_{\beta}}{\omega^{1-\alpha}}
$$

It is important to note that there is significant ongoing effort to build fractional-order capacitors with constant (yet tunable) values of the dispersion coefficient $\alpha$ and pseudo-capacitance $C_{\alpha}$ over a wide frequency range for various applications particularly in industrial automation [4] and biology [5], [6]. In [7] graphene-percolated polymer composite was used for manufacturing a fractional-order capacitor while in [8] and [9] other metal-liquid interface techniques were reported. However, for systems on chip, fully integrated solutions are required. Test results of a chip containing fabricated CMOS fractional-order capacitor emulators were recently reported in [10]. To-date, realization of a fractional-order inductor has

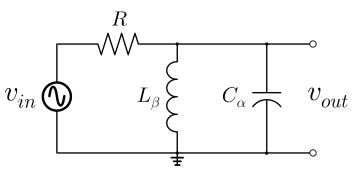

Fig. 1. Fractional-order parallel resonance network.

only been approached through the realization of a fractionalorder capacitor using appropriately configured RC networks [11] and then through the employment of a Generalized Impedance Converter (GIC) to transform it into an inductor 
[12], [13], [14], [15], [16] or using discrete Current Feedback Operational Amplifiers (CFOAs) as active elements [17].

The main contribution of the work presented is that, according to the best authors' knowledge, it is the first time in the literature where a fully integrated electronically tunable CMOS fractional inductor emulator is fabricated and experimentally verified. Having also available CMOS electronically tunable CPE emulators [10], it is possible to design a fully tunable CMOS version of the circuit in Fig. 1. The complete design is based on current-controlled transconductance amplifier cells ( $g_{m}$-cells) as detailed in [18]. In Section 2, we derive important characteristics of the fractional-order parallel resonance circuit similar to those reported in [19] for a series resonance network. In Section 3, the concept of emulating a fractional-order inductor is briefly described and experimental results from a fabricated chip in AMS $0.35 \mu \mathrm{m}$ CMOS technology are shown. In Section 4, the fully integrated parallel resonator performance is evaluated. Since our emulator is optimized for low frequency operation, very large inductance values (typically in kHenry) are required.

\section{Fractional-ORder PARALlEL RESONANCE}

The transfer function of the circuit in Fig. 1 is given by

$$
H(s)=\frac{\frac{1}{R C_{\alpha}} s^{\beta}}{s^{(\alpha+\beta)}+\frac{1}{R C_{\alpha}} s^{\beta}+\frac{1}{L_{\beta} C_{\alpha}}}
$$

and $B_{p}=1 / R C_{\alpha}$ is the pseudo-bandwidth while $\omega_{p r}=$ $1 / \sqrt{L_{\beta} C_{\alpha}}$ is the pseudo-resonance frequency both of which can be expressed in proper $\mathrm{rad} / \mathrm{sec}$ units using (3) and (4) as

$$
B_{p}=(1 / R C)^{1 /(2-\alpha)} \text { and } \omega_{p r}=(1 / L C)^{1 /(4-\alpha-\beta)}
$$

Note that the actual resonance frequency $\omega_{r}$ is obtained by solving for $\omega$ the equation $\operatorname{Im}\{H(j \omega)\}=0$ which yields

$$
\omega_{r}=\left(\omega_{p r}^{2}\right)^{\frac{1}{(\alpha+\beta)}}=\left(\frac{1}{L_{\beta} C_{\alpha}}\right)^{\frac{1}{(\alpha+\beta)}}=\frac{1}{\sqrt{L C}}
$$

where $L$ and $C$ are as given by (5). Also note that at $\omega_{r}$, $\angle H\left(j \omega_{r}\right)$ exhibits a $\pm \pi$ phase change [19]. Meanwhile, the frequency at which the magnitude response of the resonance network is maximum is denoted as $\omega_{p k}$ and is obtained by solving for $\omega$ the equation $d|H(j \omega)| / d \omega=0$ which yields

$$
\begin{gathered}
\frac{\omega_{p k}^{2(\alpha+\beta)}+B_{p} \cdot \omega_{p k}^{(\alpha+2 \beta)} \cos \left(\frac{\alpha \pi}{2}\right)}{(\beta / \alpha) \omega_{p r}^{2}}=\omega_{p r}^{2}+ \\
B_{p} \cdot \omega_{p k}^{\beta} \cos \left(\frac{\beta \pi}{2}\right)+\left(1-\frac{\alpha}{\beta}\right) \omega_{p k}^{(\alpha+\beta)} \cos \left(\frac{(\alpha+\beta) \pi}{2}\right)
\end{gathered}
$$

Note that $\omega_{p k}$ is not equal in general to $\omega_{p r}$ or $\omega_{r}$ which means that the peak in magnitude does not necessarily happen at the pseudo-resonance or resonance frequencies. However, only for $\alpha=\beta=1$ (9) yields

$$
\omega_{p k}^{4}=\omega_{p r}^{4} \rightarrow \omega_{p k}=\omega_{p r}
$$

Recalling from (8) that in this case $\omega_{r}=\omega_{p r}=1 / \sqrt{L C}$ indicates that the magnitude peaking frequency will coincide with the resonance frequency only if $\alpha=\beta=1$.

\section{Electronically-Tunable Fractional-Order INDUCTOR}

To achieve a fully tunable emulator of Fig. 1, we consider building an emulator for the fractional-order inductor.

\section{A. Emulator Architecture}

The functional block diagram for emulating a fractionalorder inductor is shown in Fig. 2 where a fractional-order

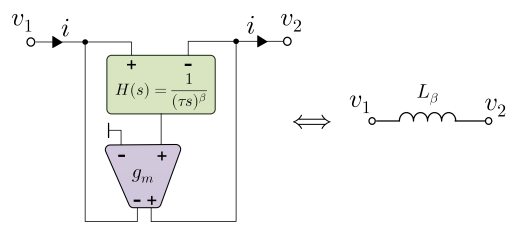

Fig. 2. Fractional-order inductor emulator.

integrator of order $\beta$ is required along with a voltage-to-current $(V \rightarrow I)$ converter. It can be shown that the realized pseudoinductance is given by

$$
L_{\beta}=\frac{1}{g_{m_{V I}} \cdot \omega_{0}^{\beta}}
$$

where $g_{m_{V I}}$ is the transconductance of the $V \rightarrow I$ converter and $\omega_{0}\left(\omega_{0}=1 / \tau_{0}\right)$ is the unity-gain frequency of the integrator. Employing the optimized $2^{n d}$-order approximation from [20], the transfer function for approximating a fractionalorder integrator around a frequency $\omega_{0}$ can be described as

$$
\frac{1}{\left(\tau_{0} s\right)^{\beta}} \cong \frac{\left(\frac{\beta^{2}-3 \beta+2}{\beta^{2}+3 \beta+2}\right) s^{2}+\left(\frac{1}{\tau_{0}} \cdot \frac{8-2 \beta^{2}}{\beta^{2}+3 \beta+2}\right) s+\frac{1}{\tau_{0}^{2}}}{s^{2}+\left(\frac{1}{\tau_{0}} \cdot \frac{8-2 \beta^{2}}{\beta^{2}+3 \beta+2}\right) s+\left(\frac{1}{\tau_{0}^{2}} \cdot \frac{\beta^{2}-3 \beta+2}{\beta^{2}+3 \beta+2}\right)}
$$

Similar to the procedure described in [18], an Inverse Followthe-Leader Feedback (IFLF) structure can be used to realize this integrator in a voltage-input voltage-output topology such that

$$
\frac{v_{\text {out }}(s)}{v_{\text {in }}(s)}=\frac{G_{2} s^{2}+\frac{G_{1}}{\tau_{1}} s+\frac{G_{0}}{\tau_{1} \tau_{2}}}{s^{2}+\frac{1}{\tau_{1}} s+\frac{1}{\tau_{1} \tau_{2}}}
$$

which can be directly compared with (12) to identify the coefficients $G_{0,1,2}$ and $\tau_{1,2}$.

\section{B. Circuit Realization}

Using Operational Transconductance Amplifiers ( $g_{m}$-cells), Fig. 2 can be implemented as shown in Fig.3(a) with each $g_{m^{-}}$ cell being realized by the enhanced linearity current-controlled topology shown in Fig. 3(b). The expression of the cell transconductance is

$$
g_{m}=\frac{5}{9} \cdot \frac{I_{\text {bias }}}{n V_{T}}
$$

where $V_{T}$ is the thermal voltage $\left(26 \mathrm{mV} @ 27^{0} \mathrm{C}\right), n$ is the subthreshold slope factor of a MOS transistor, and $I_{b i a s}$ is the bias current [21].

Using (5) and (11) it is obtained that at $\omega=\omega_{0}$ the impedance of the fractional-order inductor will be equal to $L \omega_{0}=L_{\beta} \omega_{0}^{\beta}=1 / g_{m V I}$. Therefore, the bias current of the 


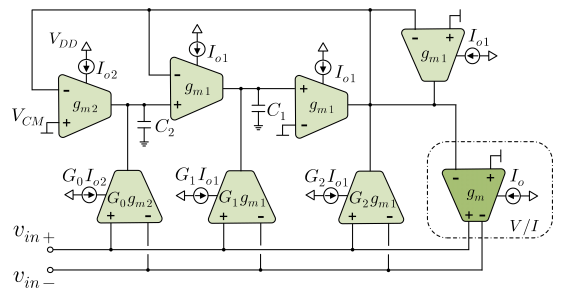

(a)

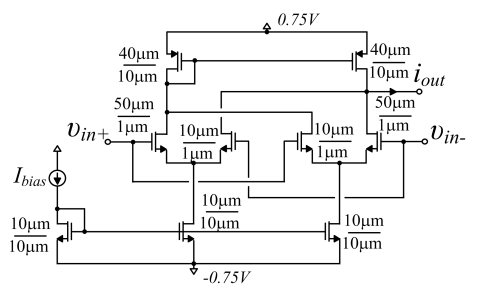

(b)

Fig. 3. Fractional-order inductor emulator circuit using (a) current-controlled $g_{m}$-cells each with the MOS realization shown in (b).

$V \rightarrow I$ converter $\left(I_{0 V I}\right)$ will be chosen according to the desired value of the inductance $L$ at a specific frequency $\omega_{0}$. Assuming capacitors with equal value, i.e $C_{1}=C_{1}=C_{e x t}$, the design equations of the inductor emulator are summarized in Table I. In order to emulate an inductance $L=1.014 \times$

TABLE I

DESIGN EQUATIONS TO ACHIEVE INDUCTANCE $L$ AT FREQUENCY $\omega_{0}$.

\begin{tabular}{|c|c|}
\hline Variable & Expression \\
\hline \hline$I_{0 V I}$ & $\frac{9}{5} \cdot \frac{n V_{T}}{L \omega_{0}}$ \\
\hline$C_{e x t}$ & $\frac{5}{9} \cdot \frac{I_{0}}{n V_{T} \omega_{0}} \cdot \frac{-2 \beta^{2}+8}{\beta^{2}-3 \beta+2}$ \\
\hline$I_{01}$ & $\frac{9}{5} \cdot \frac{-2 \beta^{2}+8}{\beta^{2}+3 \beta+2} \cdot n V_{T} C_{e x t} \omega_{0}$ \\
\hline$I_{02}$ & $\frac{9}{5} \cdot \frac{\beta^{2}-3 \beta+2}{-2 \beta^{2}+8} \cdot n V_{T} C_{e x t} \omega_{0}$ \\
\hline$G_{2}$ & $\frac{\beta^{2}-3 \beta+2}{\beta^{2}+3 \beta+2}$ \\
\hline$G_{1}$ & 1 \\
\hline$G_{0}$ & $\frac{\beta^{2}+3 \beta+2}{\beta^{2}-3 \beta+2}$ \\
\hline
\end{tabular}

$10^{3} H @ 100 H z$ for $\beta=(0.2,0.5,0.8)$ or, equivalently, pseudoinductances $L_{\beta}=\left(175 \times 10^{3}, 25.4 \times 10^{3}, 3.7 \times 10^{3}\right) \mathrm{H} / \mathrm{sec}^{1-\beta}$, the calculated values are given in Table II.

Inspecting Table I, the following conclusions are derived with regards to the electronic tunability of the proposed scheme: (a) the value of the inductance at a specific frequency could be electronically tuned through the the bias current of the $V \rightarrow I$ converter $\left(I_{0 V I}\right)$; if this current will be tuned into a new value $I_{o V I}^{\prime}=m \cdot I_{0 V I}$, then the value of the inductance at specific frequency $\omega_{0}$ becomes equal to $L^{\prime}=L / m$. Note from (5) that the pseudo-inductance $L_{\beta}$ will be also scaled by the same factor, (b) the unity-gain frequency of the integrator $\left(\omega_{0}\right)$ can be electronically tuned through the dc bias currents $I_{01}$ and $I_{02}$. Thus, if these currents will be scaled by a factor $m$, then $\omega_{0}$ will be scaled by the same factor. It should be mentioned at this point that linear scaling is possible in the value of the core integrator unity gain frequency $\omega_{0}$ only. Other critical frequencies of the resonance circuit, such as $\omega_{r}, \omega_{p r}, \omega_{p k}$ do not follow a linear scaling.
In our design, a main bias current $I_{0}=I_{0 V I}$ has been utilized, while the bias currents $I_{0 i}(i=1,2)$ as well as their scaled versions $G_{j} I_{0 i},(j=0,1,2)$ have been implemented by appropriately configured current-mirror stages. Thus, scaling the supply current $I_{0}$ by a factor $m$ such that the new value $I_{o}^{\prime}$ is given by the expression: $I_{o}^{\prime}=m \cdot I_{0}$ reflects to the following: (a) the bias currents $I_{0 i}(i=1,2)$ will be changed by the same factor and, therefore, the unity-gain frequency of the integrator becomes: $\omega_{0}^{\prime}=m \omega_{0}$, (b) the bias current of the $V \rightarrow I$ converter will also be changed by the same factor and the values of $L$ at $\omega_{0}^{\prime}$ and $L_{\beta}$ will be changed as $L^{\prime}=L / m^{2}$ and $L_{\beta}^{\prime}=L_{\beta} / m^{1+\beta}$, respectively.

TABLE II

Design VAlues For Fig. 3(A) To ACHIEVe $L=1.014 \times 10^{3} \mathrm{H} @ 100 \mathrm{~Hz}$.

\begin{tabular}{|c|c|c|c|}
\hline Variable & $\beta=0.2$ & $\beta=0.5$ & $\beta=0.8$ \\
\hline \hline$I_{0 V I}$ & $95 n A$ & $95 n A$ & $95 n A$ \\
\hline$C_{e x t}$ & $13.8 n F$ & $25 n F$ & $70 n F$ \\
\hline$I_{01}$ & $1.58 \mu A$ & $1.91 \mu A$ & $3.57 \mu A$ \\
\hline$I_{02}$ & $95 n A$ & $95 n A$ & $95 n A$ \\
\hline$G_{2}$ & 0.545 & 0.2 & 0.048 \\
\hline$G_{1}$ & 1 & 1 & 1 \\
\hline$G_{0}$ & 1.83 & 5 & 21 \\
\hline
\end{tabular}

\section{Chip Fabrication and Test Results}

A chip containing three fractional-order inductors of orders $\beta=0.2,0.5,0.8$ with $L=1.014 \times 10^{3} \mathrm{H} @ 100 \mathrm{~Hz}$ along side three fractional-order capacitors of similar orders was fabricated using an AMS $0.35 \mu \mathrm{m}$ C35B 4 CMOS process. The die photo is shown in Fig. 4(a), where each inductor emulator measures $236 \mu \mathrm{m} \times 225 \mu \mathrm{m}$, while the whole die measured $1.68 \mathrm{~mm} \times 1.68 \mathrm{~mm}$. The designed inductors were tested using the HIOKI 3522 HiTESTER LCR meter and screen shots are demonstrated in Fig. 4(b). Applying a differential input voltage of $20 \mathrm{mV}$ and employing $V_{C M}=0 \mathrm{~V}$ and a main bias current $I_{0}=95 n \mathrm{~A}$, the measured magnitude measurements are demonstrated in Fig. 5(a).

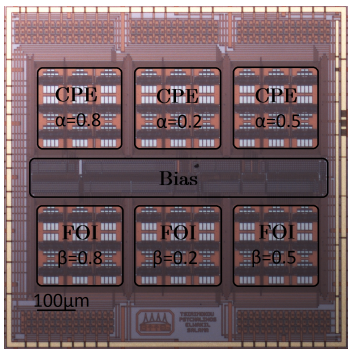

(a)

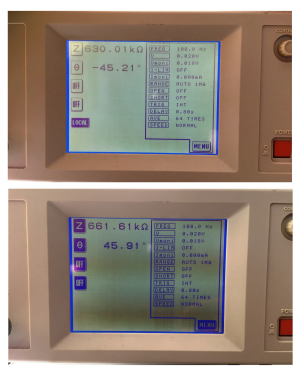

(b)
Fig. 4. (a) Die photo of the fabricated chip containing fractional-order inductors and capacitors, and (b) screen shots from the LCR tester for fractional-order capacitor/inductor.

The measured pseudo-inductances were calculated as $L_{\beta}=\left(164 \times 10^{3}, 25.4 \times 10^{3}, 4.1 \times 10^{3}\right)$ Henry $/ \mathrm{sec}^{1-\beta}$, respectively. In addition, Fig. 5(b) shows the measured impedance phases compared with the theoretically predicted 


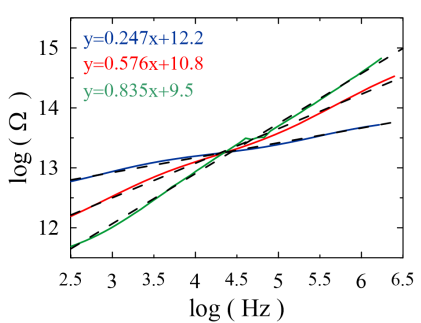

(a)

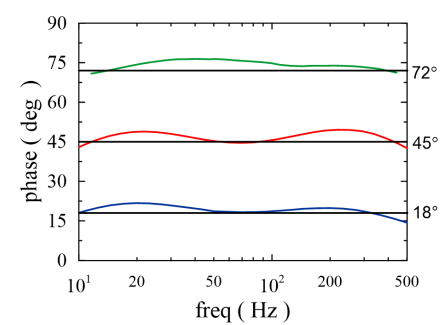

(b)
Fig. 5. Experimental (a) magnitude and (b) phase responses for the fabricated fractionalorder inductors at $95 n \mathrm{~A}$ bias current.

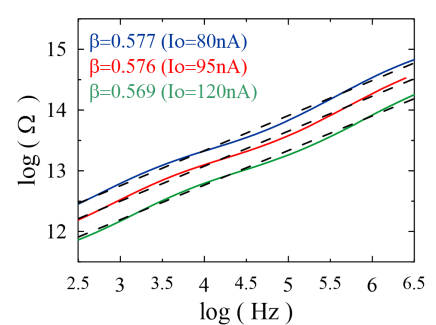

(a)

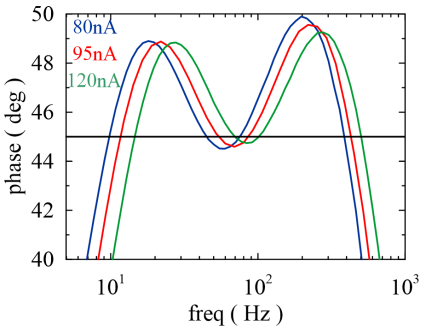

(b)
Fig. 6. Demonstration of the tunability of the fractional-order inductor (a) magnitude and (b) phase $(80,95,120) n A$ bias currents.

values $\left(18^{0}, 45^{0}, 72^{0}\right)$, respectively. Due to the limitations of the $2^{\text {nd }}$-order approximation particularly as the fractional order increases, the phase and magnitude error increase beyond $700 \mathrm{~Hz}$. However, in the range $10 \mathrm{~Hz}-700 \mathrm{~Hz}$, the phase and magnitude errors do not exceed $10 \%$ for all emulators. The investigation of tunability for the bias currents $80 n A$, $95 n A$ and $120 n A$ for a fixed order $\beta=0.5$ is demonstrated in Fig. 6. The measured pseudo-inductances were $L_{\beta}=\left(31.24 \times 10^{3}, 25.42 \times 10^{3}, 18.91 \times 10^{3}\right)$ Henry $/ \mathrm{sec}^{0.5}$, which are very close to the theoretically predicted values $\left(31.13 \times 10^{3}, 25.4 \times 10^{3}, 18.03 \times 10^{3}\right)$ Henry/ $\mathrm{sec}^{0.5}$. In addition, both magnitude and phase errors remain at the same level (i.e. $<10 \%$ ) in these plots.

\section{Fractional-ORder PARALlel Resonator EMULATOR}

As a first step, the impedance of the parallel $L_{\beta} C_{\alpha}$ resonator is tested employing a bias current equal to $95 \mathrm{nA}$. Thus, selecting a center frequency $f_{0}=100 \mathrm{~Hz}$, the resonator was tested for $C_{\alpha}=(433,62.6,9.07) n$ Farad $/ \sec ^{1-a}$ and $L_{\beta}=\left(175.6 \times 10^{3}, 25.4 \times 10^{3}, 3.7 \times 10^{3}\right)$ Henry $/ \mathrm{sec}^{1-\beta}$, for orders $0.2,0.5$, and 0.8 , respectively. It should be stressed at this point that the topology in Fig. 3(a) is also capable for emulating a fractional-order capacitor, just by choosing appropriate values for capacitors and biasing currents and this is an attractive benefit offered by the proposed concept [18]. The design values for emulating pseudo-capacitance $C_{\alpha}=(433,62.6,9.07) n$ Farad $/ \mathrm{sec}^{1-\alpha}$, i.e capacitance $C=2.5 n F @ 100 H z$, for $\alpha=(0.2,0.5,0.8)$ are summarized in Table III. Applying a differential input signal $20 \mathrm{mV}$, the magnitude and phase responses are shown in Fig. 7. The obtained frequency characteristics are summarized in Table IV, where the corresponding theoretically predicted values are also given in parentheses. Note that the $Q$ factor is not measurable for $\alpha=0.5, \beta=0.2$ because the theoretical lower cutoff frequency is about $2 \mathrm{~Hz}$ which is outside the limits of the employed approximation. With regards to the magnitude and phase errors, in the case of resonator, they do not exceed a $10 \%$ level in the range $20 \mathrm{~Hz}-900 \mathrm{~Hz}$.

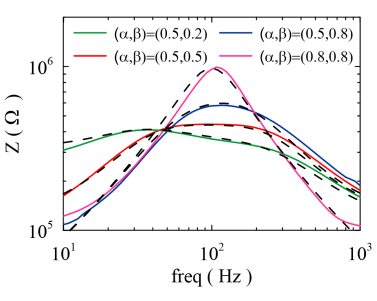

(a)

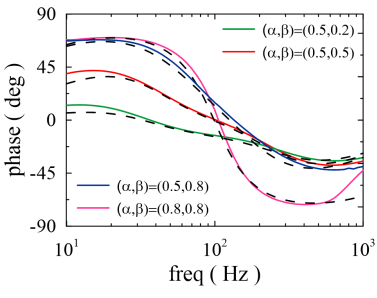

(b)
Fig. 7. Impedance measurements of (a) magnitude and (b) phase of parallel resonance $L_{\beta} C_{a}$ at $I_{o}=95 n A$.

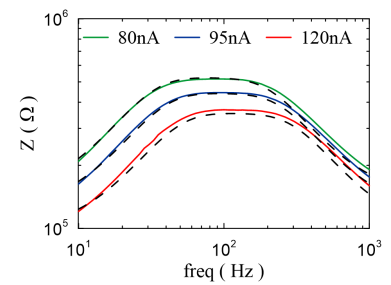

(a)

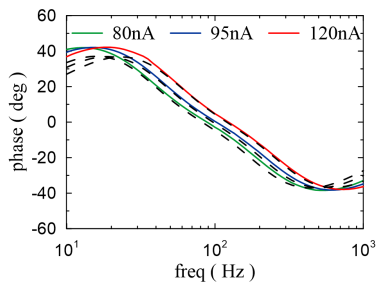

(b)
Fig. 8. Demonstration of the tuning capability of the (a) magnitude and (b) phase of parallel resonance $L_{0.5} C_{0.5}$ at $(80,95,120) n A$ bias currents.

TABLE III

DESIGN VALUES FOR FIG. 3 TO ACHIEVE $C=2.5 n F @ 100 H z$.

\begin{tabular}{|c|c|c|c|}
\hline Variable & $\alpha=0.2$ & $\alpha=0.5$ & $\alpha=0.8$ \\
\hline \hline$I_{0 V I}$ & $95 n A$ & $95 n A$ & $95 n A$ \\
\hline$C_{\text {ext }}$ & $7.5 n F$ & $5 n F$ & $3.3 n F$ \\
\hline$I_{01}$ & $1.58 \mu A$ & $1.91 \mu A$ & $3.57 \mu A$ \\
\hline$I_{02}$ & $95 n A$ & $95 n A$ & $95 n A$ \\
\hline$G_{2}$ & 1.83 & 5 & 21 \\
\hline$G_{1}$ & 1 & 1 & 1 \\
\hline$G_{0}$ & 0.545 & 0.2 & 0.048 \\
\hline
\end{tabular}

Concerning the tunability of the resonator, the magnitude and phase responses for fixed orders $\alpha=\beta=0.5$ are demonstrated in Fig. 8. The obtained results are summarized in Table $\mathrm{V}$, where the corresponding theoretically predicted values are also given in parentheses.

After that, the resonator circuit of Fig.1, which is essentially a fractional-order bandpass filter, was tested using different values of the resistor and the already tested $L_{\beta} C_{\alpha}$ setup. The obtained responses of the fractional-order bandpass filter for $\alpha=\beta=0.5$ and bias current $I_{o}=95 n A$ are demonstrated, 


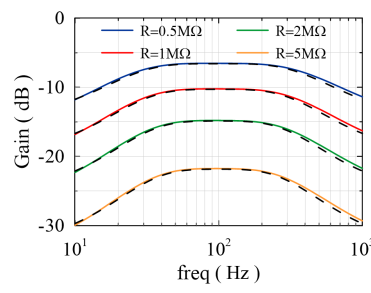

(a)

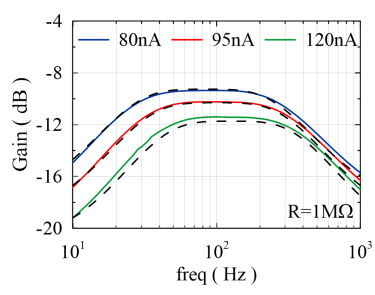

(b)
Fig. 9. Fractional-order bandpass filter responses $(\alpha=\beta=0.5$ ) (a) $R=$ $(0.5,1,2,5) M \Omega, I_{o}=95 n A$, and (b) $R=1 M \Omega, I_{o}=(80,95,120) n A$.

TABLE IV

Performance CHARACTERISTICS OF THE $L_{\beta} C_{\alpha}$ Resonator.

\begin{tabular}{|c|c|c|c|c|}
\hline Parameter & $(0.5,0.2)$ & $(0.5,0.5)$ & $(0.5,0.8)$ & $(0.8,0.8)$ \\
\hline \hline$f_{p k}(H z)$ & $36.7(38.2)$ & $92.84(100)$ & $116(121.3)$ & $107.7(100)$ \\
\hline$Q$ factor & - & $0.25(0.29)$ & $0.44(0.52)$ & $1.19(1.11)$ \\
\hline$Z @ f_{p k}(k \Omega)$ & $411(409)$ & $444(440)$ & $580(601)$ & $992(985)$ \\
\hline$f_{p r}(H z)$ & $38.1(33.4)$ & $103(100)$ & $134(130)$ & $105(100)$ \\
\hline
\end{tabular}

along with the corresponding theoretically predicted responses, in Fig. 9(a). The corresponding plots for $R=1 M \Omega$ and $I_{o}=(80,95,120) n A$ are provided in Fig. $9(\mathrm{~b})$, to confirm the electronic tuning capability of the bandpass filter.

\section{CONCLUSIONS}

A fully integrated fractional-order resonance emulator circuit capable of operating in the low frequency range of $10 \mathrm{~Hz}-700 \mathrm{~Hz}$ was proposed. The circuit shows good accuracy in this frequency range when compared to theoretical values and is tunable via a bias current. Although it is possible to also emulate a fractional-order resonance circuit on a Field Programmable Analog Array (FPAA) platform [20], low frequency operation will be difficult to achieve due to the very large values of inductance which are impossible to realize with the FPAA switched-capacitor technology. The same holds for the fractional-order parallel resonance circuit recently reported in [15] which is in discrete IC component form and relies on GIC to transform the fractional-order capacitor into a fractional-order inductor.

\section{ACKNOWLEDGMENT}

This work was supported by Grant E.029 from the Research Committee of the University of Patras (Programme K. Karatheodori).

\section{REFERENCES}

[1] A. S. Elwakil, "Fractional-order circuits and systems: An emerging interdisciplinary research area," IEEE Circuits and Systems Magazine, vol. 10 , no. 4, pp. 40-50, 2010.

[2] S. Westerlund and L. Ekstam, "Capacitor theory," IEEE Trans. on Dielectrics and Electrical Insulation, vol. 1, no. 5, pp. 826-839, 1994.

[3] A. S. Elwakil, A. Allagui, B. J. Maundy, and C. Psychalinos, "Low frequency oscillator using a super-capacitor," Int. J. Electronics and Communications (AEU), vol. 70, no. 7, pp. 970-973, July 2016.

[4] M. O. Efe, "Fractional order systems in industrial automation - a survey," IEEE Transactions on Industrial Informatics, vol. 7, no. 4, pp. 582-591, 2011.
TABLE V

TUNABility OF THE $L_{\beta} C_{\alpha}$ RESONATOR AT $(80,95,120) n A$ BIAS CURRENTS

\begin{tabular}{|c|c|c|c|}
\hline Parameter $(\alpha=\beta=0.5)$ & $I_{o}=80 n A$ & $I_{o}=95 n A$ & $I_{o}=120 n A$ \\
\hline \hline$f_{p k}(H z)$ & $80(83.69)$ & $92.84(100)$ & $100(125.3)$ \\
\hline$Z @ f_{p k}(k \Omega)$ & $516(525)$ & $444(440)$ & $368(350)$ \\
\hline$f_{p r}(H z)$ & $89.5(83.5)$ & $103(100)$ & $123(125.3)$ \\
\hline
\end{tabular}

[5] R. L. Magin, Fractional Calculus in Bioengineering. Begell House Redding, 2006

[6] K. Moaddy, A. G. Radwan, K. N. Salama, S. Momani, and I. Hashim, "The fractional-order modeling and synchronization of electrically coupled neuron systems," Computers \& Mathematics with Applications, vol. 64, no. 10, pp. 3329-3339, 2012.

[7] A. M. Elshurafa, M. N. Almadhoun, K. N. Salama, and H. N. Alshareef, "Microscale electrostatic fractional capacitors using reduced graphene oxide percolated polymer composites," Applied Physics Letters, vol. 102, no. 23, p. 232901, 2013.

[8] I. S. Jesus and J. A. Tenreiro-Machado, "Development of fractional order capacitors based on electrolyte processes," Nonlinear Dynamics, vol. 56, no. 1-2, pp. 45-55, 2009.

[9] M. Sivaramakrishna, S. Das, K. Biswas, and B. Goswami, "Fabrication of a fractional order capacitor with desired specifications: A study on process identification and characterization," IEEE Trans. on Electron Devices, vol. 58, pp. 4067-4073, 2011.

[10] G. Tsirimokou, C. Psychalinos, A. S. Elwakil, and K. N. Salama, "Experimental verification of on-chip CMOS fractional-order capacitor emulators," Electronics Letters, vol. 52, no. 15, pp. 1298-1300, July 2016.

[11] G. L. Abulencia and A. C. Abad, "Analog realization of a low-voltage two-order selectable fractional-order differentiator in a 0.35 um CMOS technology," in Proc. IEEE International Conference on Humanoid, Nanotechnology, Information Technology, Communication and Control, Environment and Management (HNICEM), Cebu City, Philippines, 2015, pp. 1-6.

[12] T. J. Freeborn, B. Maundy, and A. S. Elwakil, "Fractional resonance-based filters," Mathematical Problems in Engineering, vol. (http://dx.doi.org/10.1155/2013/726721, 2013.

[13] A. G. Radwan and M. E. Fouda, "Optimization of fractional-order RLC filters," Circuits, Systems, and Signal Processing, vol. 32, no. 5, pp. 2097-2118, 2013.

[14] M. C. Tripathy, D. Mondal, K. Biswas, and S. Sen, "Experimental studies on realization of fractional inductors and fractional-order bandpass filters," International Journal of Circuit Theory and Applications, vol. 43, no. 9, pp. 1183-1196, 2015.

[15] A. Adhikary, S. Sen, and K. Biswas, "Practical realization of tunable fractional order parallel resonator and fractional order filters," IEEE Transactions on Circuits and Systems I: Fundamental Theory and Applications, vol. 63, no. 8, pp. 1142-1151, 2016.

[16] A. S. Ali, A. Radwan, and A. M. Soliman, "Fractional-order Butterworth filter: Active and passive realizations," IEEE Journal on Emerging and Selected Topics in Circuits and Systems, vol. 3, no. 3, pp. 346-354, 2013.

[17] I. Dimeas, G. Tsirimokou, C. Psychalinos, and A. Elwakil, "Experimental verification of filters using fractional-order capacitor and inductor emulators," in 39th International Conference on Telecommunications and Signal Processing (TSP), Vienna, Austria, June 2016, pp. 559-562.

[18] G. Tsirimokou, C. Psychalinos, and A. S. Elwakil, "Emulation of a constant phase element using operational transconductance amplifiers," Analog Integrated Circuits and Signal Processing, vol. 85, no. 3, pp. 413-423, 2015.

[19] A. G. Radwan and K. N. Salama, "Passive and active elements using fractional LC circuit," IEEE Transactions on Circuits and Systems I: Regular Papers, vol. 58, no. 10, pp. 2388-2397, 2011.

[20] T. J. Freeborn, B. Maundy, and A. S. Elwakil, "Field programmable analogue array implementation of fractional step filters," IET Circuits, Devices \& Systems, vol. 4, no. 6, pp. 514-524, 2010.

[21] P. Corbishley and E. Rodriguez-Villegas, "A nanopower bandpass filter for detection of an acoustic signal in a wearable breathing detector," IEEE Transactions on Biomedical Circuits and Systems, vol. 1, no. 3, pp. 163-171, 2007. 